\title{
Abordagem e tratamento de úlcera de pressão infectada em idosa sob cuidado domiciliar: da atenção primária à especializada
}

\author{
Approach and treatment of infected pressure ulcer in the elderly under home care: from primary to \\ specialized care
}

Maria Júlia Campos Guerra**; Júlia Pereira Alvim; Lucas Oliveira Salles†; Roberta Maria Rola Albergaria†; José Carlos Dantas Teixeira*; Aline Trovão Queiroz

Como citar esse artigo. Guerra, M.J.C.;

Alvim, J.P.; Salles, L.O.; Albergaria

R.M.R.; Teixeira J.C.D.; Queiroz,

A.T. Abordagem e tratamento de

úlcera de pressão infectada em idosa sob cuidado domiciliar: da atenção primária à especializada. Revista de Saúde. 2021 Dez./Mar.; 12 (1): 30-34.

\section{Resumo}

Nos últimos anos, a população idosa vem aumentando no Brasil e tal fato é acompanhado pelo surgimento de comorbidades, dentre as quais a úlcera por pressão (UP), uma afecção de etiologia multifatorial, considerada problema de saúde pública. Nesse contexto, o cuidado domiciliar prestado pela família do doente, com apoio da equipe da Estratégia de Saúde da Família (ESF), se apresenta como tática capaz de responder à necessidade real da pessoa com condições crônicas. No âmbito da Atenção Primária à Saúde (APS), a equipe da ESF, visando garantir a integralidade do cuidado, é responsável por referenciar, quando necessário, o paciente à Atenção Secundária. Esse artigo objetiva relatar o caso de uma idosa com fatores de risco para UP, que apresentou uma UP em calcâneo infectada por míase, sendo referenciada ao serviço de Cirurgia Geral de um Hospital Universitário para desbridamento da lesão e, posteriormente, retornando à APS para continuidade do cuidado. Os dados deste relato foram colhidos do prontuário da paciente em um Hospital Universitário. Para revisão e discussão deste artigo foram utilizadas as bases de dados Lilacs, Scielo, Medline, BVS e Pubmed. Ressaltase a importância do cuidado integral, buscando garantir a promoção, proteção, recuperação, reabilitação e manutenção da saúde do paciente. Desse modo, os cuidadores familiares, orientados por profissionais de saúde, são também atores essenciais na continuidade do cuidado, pois assim, aliando práticas curativas às preventivas, em todos os níveis de atenção à saúde, busca-se uma melhor qualidade de vida para o paciente.

Palavras-chave: Lesão por Pressão; Assistência Domiciliar; Atenção Primária à Saúde; Integralidade em Saúde; Atenção Secundária à Saúde.

\begin{abstract}
In recent years, the elderly population has been increasing in Brazil, and this is accompanied by the emergence of comorbidities, including pressure ulcer (PU), a condition of multifactorial etiology, considered public health problem. In this context, home care provided by the patient's family, with the support of the Family Health Strategy (ESF) team, presents itself as a tactic capable of responding to the real need of the person with chronic conditions. In the scope of Primary Health Care (PHC), the ESF team, aiming to guarantee the integrality of care, is responsible for referring, when necessary, the patient to Secondary Care. This article objetive is report the case of an elderly woman with risk factors for PU, who presented a PU in calcaneus infected by myiasis, being referred to the General Surgery service of a University Hospital for debridement of the lesion and, later, returning to the APS for continuity of the caution. Data from this report were collected from the patient's chart at a University Hospital. For the review and discussion of this article, Lilacs, Scielo, Medline, BVS and Pubmed databases were used. The importance of integral care is emphasized, seeking to guarantee the promotion, protection, recovery, rehabilitation and maintenance of the patient's health. In this way, family caregivers, guided by health professionals, are also essential actors in the continuity of care, since, combining preventive and curative practices at all levels of health care, a better quality of life is sought for the patient.

Keywords: Pressure Ulcer; Home Nursing; Primary Health Care; Integrality in Health; Secondary Care.
\end{abstract}

\section{Introdução}

Os avanços da medicina, ao longo dos últimos anos, possibilitaram notório aumento da expectativa de vida, resultando no crescimento da população idosa. A Organização Mundial da Saúde (OMS) estima que em 2050 haverá 2 bilhões de idosos no mundo ${ }^{1}$.

As transformações corporais próprias do envelhecimento podem acarretar vulnerabilidade a traumas, redução da massa muscular, aumento de comorbidades, redução de atividade e de mobilidade ${ }^{2}$. Nesse contexto, observa-se o aumento da incidência de casos de úlcera por pressão (UP), tornando essa um problema de saúde pública.

O desenvolvimento da UP representa um importante impacto financeiro para as instituições, sendo essa também considerada um problema socioeconômico e educacional. Por esse motivo, o investimento na prevenção, cujos custos são inferiores ao tratamento, é primordial. O cuidado das UPs ocupa o terceiro lugar

¥ Docente do Curso de Medicina da Universidade de Vassouras, Vassouras, Rio de Janeiro, Brasil 
em gastos em saúde, sendo ultrapassado somente pelos gastos com tratamento de câncer e cirurgia cardíaca ${ }^{3}$.

A UP pode ser definida como uma lesão na pele e/ou no tecido subjacente de etiologia multifatorial, normalmente sobre uma proeminência óssea, em resultado da pressão ou de uma combinação entre essa e forças de cisalhamento e fricção. As localizações mais frequentes são: isquiática (24\%), sacrococcígea (23\%), trocantérica $(15 \%)$ e calcânea $(8 \%)^{1}$.

A mobilidade física diminuída é considerada o fator de maior importância no desenvolvimento da UP, pois afeta a capacidade de aliviar a pressão de modo eficaz ${ }^{4}$. Dentre os outros fatores de risco, estão: idade avançada, obesidade, limitação nas atividades de vida diária, incontinência urinária e/ou anal, anemia, infecção e estado nutricional ${ }^{5}$. Além desses, certas doenças predispõem ao desenvolvimento da UP por tornar os pacientes acamados, como Alzheimer e sequela de acidente vascular encefálico.

$\mathrm{O}$ uso de medicamentos, como analgésicos e sedativos, por alterarem a mobilidade e o estímulo doloroso normal, e os anti-hipertensivos, que reduzem o fluxo sanguíneo e a perfusão tissular, também são citados como fatores predisponentes para o desenvolvimento da UP. E ainda, outro fator destacado é a hipertensão arterial, em geral associada com outras desordens metabólicas, como diabetes mellitus. A hipertensão arterial aumenta a resistência vascular, ocorrendo juntamente uma hipertrofia celular da camada muscular (túnica média), a qual promove uma isquemia e hipóxia celular, facilitando o surgimento da $\mathrm{UP}^{4}$.

A literatura descreve algumas medidas preventivas que podem ser aplicadas para evitar o desenvolvimento da UP, como usar lençóis adequados, manter a pele limpa e hidratada, utilizar redistribuidores de pressão3 e, principalmente, proporcionar ao paciente um ambiente salubre e com boas condições de higiene para sua recuperação. Quando esse cenário não é viável, complicações maiores podem ocorrer, como a infecção e contaminação da ferida em casos avançados, retardando o processo de cicatrização e melhora da ferida.

Nesse contexto, diante da necessidade de uma abordagem individualizada ao paciente com UP, a Estratégia de Saúde da Família (ESF), no âmbito da Atenção Primária à Saúde (APS), exerce um papel fundamental, possibilitando um cuidado continuado ao paciente, principalmente aos que se encontram sob cuidado domiciliar, e garantindo a integralidade do cuidado, em todos os níveis de atenção à saúde.

O presente artigo objetiva relatar o caso de uma idosa portadora de hipertensão arterial sistêmica, com sequela de acidente vascular encefálico, com mobilidade reduzida, que apresentou uma UP após alta hospitalar, infectada com larvas de mí́ase, cuja abordagem e tratamento envolveu os níveis de atenção primária e secundária.
Este relato foi submetido ao Comitê de Ética e Pesquisa em Seres Humanos da Universidade de Vassouras e aprovado sob o parecer de número 3.208.373, CAAE 08287019.5.0000.5290.

\section{Relato do caso}

Paciente 72 anos, sexo feminino, negra, portadora de hipertensão arterial sistêmica e de artrose bilateral nos joelhos, com dificuldade de deambulação, usuária de cadeira de rodas, permaneceu 05 dias internada na Unidade de Terapia Intensica e 04 dias internada na enfermaria em um Hospital Universitário, após um quadro de acidente vascular encefálico, o qual deixou sequelas neurológicas, seguido de pneumonia.

No quarto dia após a alta hospitalar, ao receber a visita domiciliar (VD) da Agente Comunitária de Saúde (ACS) da ESF do seu bairro, foi constatada a presença de uma UP em região calcânea direita. No dia seguinte, acompanhada da enfermeira e do médico da ESF, foi feita nova VD à paciente, e foram feitas orientações aos seus familiares, já que esses se revezavam no cuidado domiciliar à idosa. A equipe da ESF orientou quanto à limpeza e higiene adequada da ferida, o uso de pomada cicatrizante local, a necessidade de curativo diário e da manutenção da salubridade na moradia.

Entretanto, mesmo com VDs realizadas semanalmente pela equipe da ESF, com reforço das orientações, os cuidados domiciliares não foram suficientes. Após aproximadamente 30 dias da alta hospitalar, devido à infecção da ferida, a paciente foi então referenciada para atenção especializada.

No serviço de Clínica Cirúrgica do Hospital Universitário, ao exame físico, a paciente apresentou varizes em membros inferiores, presença de intensa necrose em calcâneo direito com enorme quantidade de larvas de moscas, causando uma miíase. Foi realizado desbridamento mecânico da ferida (Figura 1) com remoção das larvas (Figura 2).

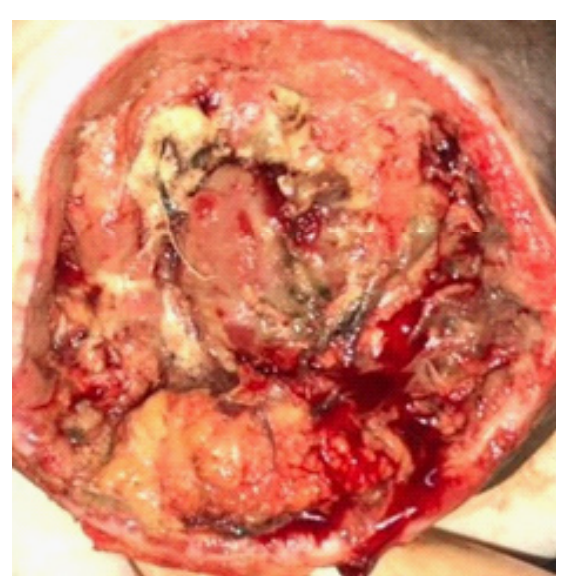

Figura 1. Desbridamento inicial da Úlcera por Pressão (UP). 


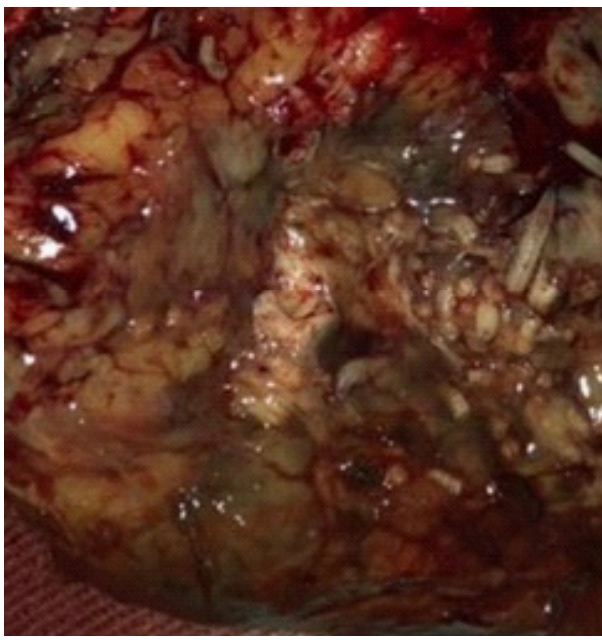

Figura 2. Remoção inicial de larvas de moscas causadoras de miíase.

Após 03 dias em uso de Colagenase na ferida, a paciente retornou ao ambulatório de Clínica Cirúrgica para reavaliação, observando-se tecido de granulação e pequena área de necrose. Dessa forma, foi realizado novo desbridamento da ferida no calcâneo (Figura 3).

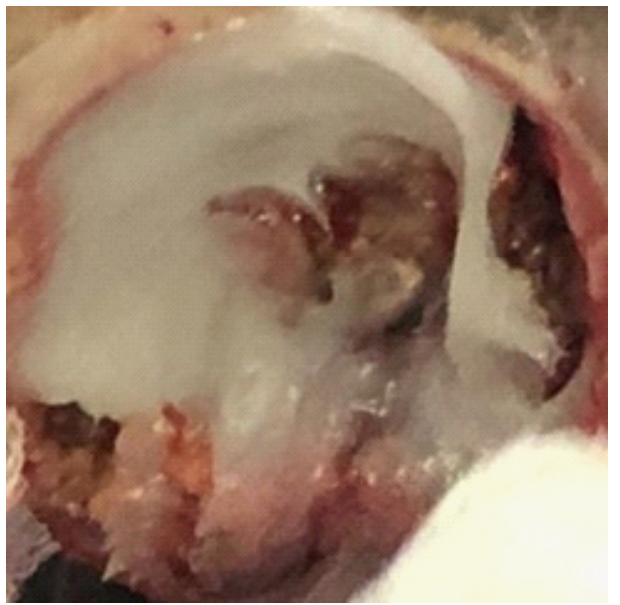

Figura 3. Repetição do desbridamento.

Após o procedimento, a paciente foi então contrareferenciada à APS para que a equipe da ESF pudesse dar continuidade ao cuidado, além de dar suporte à família no cuidado domiciliar. A equipe novamente orientou a família quanto à lavagem diária da ferida com água e sabão, uso de Colagenase pomada no local, óleo de girassol e curativo oclusivo, diariamente. Após 03 meses de monitoramento semanal da ferida pela equipe, com VDs realizadas quase que diariamente pela ACS, e intenso comprometimento dos cuidadores famíliares da paciente, a lesão apresentou melhora significativa após 02 meses do desbridamento (Figura 4), com excelente cicatrização após 03 meses do desbridamento (Figura $5)$.

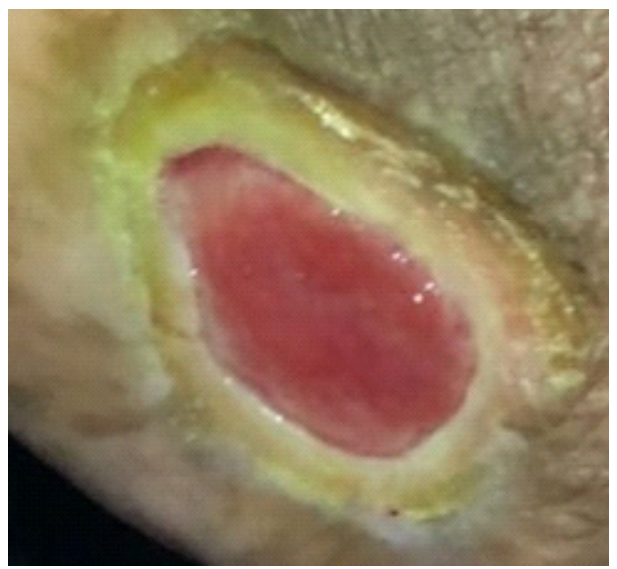

Figura 4. Cicatrização após dois meses do desbridamento.

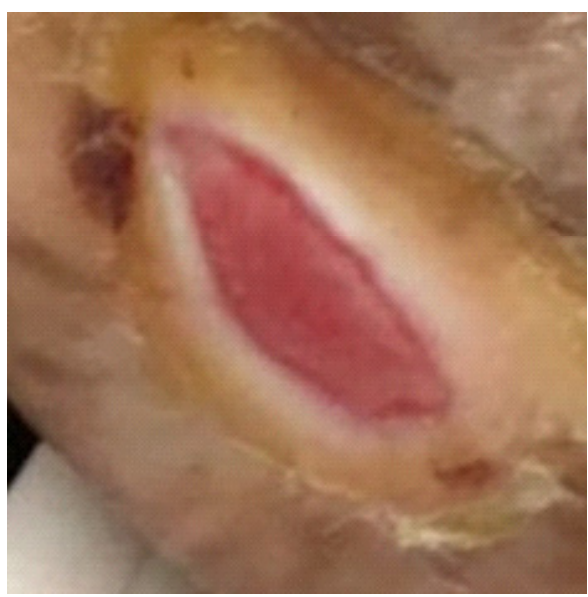

Figura 5. Cicatização após três meses do desbridamento.

\section{Discussão}

O envelhecimento traz consigo alterações na pele, como a renovação epidérmica mais lenta, a diminuição da produção do colágeno e da elasticidade, a redistribuição da gordura subcutânea para regiões mais profundas, dentre outras. Tais alterações levam à uma maior exposição das proeminências ósseas, e a capacidade do tecido de distribuir pressão tornase reduzida, assim como a vascularização ${ }^{1}$. Como consequência, aumentam os riscos de desenvolver uma UP, cuja cicatrização também torna-se mais lenta ${ }^{1}$.

Alguns autores sugerem que há uma tendência linear entre o aumento de idade e o maior risco para UP, que ocorre principalmente entre os idosos com mais de 80 anos de idade, que são pacientes que tendem a um declíneo da mobilidade física3. Corroborando com a literatura, o presente caso relatado trata-se de uma UP em idosa com mobilidade reduzida, usuária de cadeira de rodas.

Bezerra e colaboradores ${ }^{6}$, em estudo realizado com pacientes acamados sob cuidado domiciliar, 
verificaram que alguns pacientes adquiriram a UP durante o seu período de internação hospitalar. $\mathrm{Na}$ maioria dos casos, os autores observaram que era o cuidador, orientado pelos enfermeiros ou técnicos de enfermagem do Hospital de referência ou, pela equipe da ESF quem realizava o curativo $^{6}$, conforme ocorrido nesse caso relatado.

Outros autores afirmam que as UP podem desenvolver-se em 24 horas ou levar até 05 dias para se manifestar1, como foi o caso da paciente relatada, que desenvolveu a UP 04 dias após a alta hospitalar. Portanto, identificar as condições predisponentes para formação da UPé de suma importância, e os profissionais de saúde responsáveis pela prevenção da UP devem estar familiarizados com os principais fatores de risco envolvidos.

No que diz respeito às ações de prevenção das UPs, destaca-se a manutenção de higiene cutânea e o exame físico cutâneo3. Assim, na prevenção e no tratamento da UP é fundamental que haja uma visão interdisciplinar em que todas as especialidades estejam envolvidas. As medidas preventivas e de educação em saúde sobre o tema são essenciais em todas as etapas do tratamento ${ }^{1}$.

No presente caso, a família da paciente foi orientada, no momento da alta hospitalar, com relação às medidas preventivas. Entretanto, quando receberam a VD da equipe da ESF, a ferida no calcâneo já estava aparente.

Em relação à infecção das UPs, sabe-se que o processo infeccioso delimita o fechamento epitelial, pois osaltos níveis bacteriológicos nos tecidos, com a lise das bactérias, acabam por secretarem exotoxinas e produzirem endotoxinas. Assim, atendência dessas toxinas é causar necrose local, interferindo na concentração de mediadores químicos necessários para a boa evolução cicatricial ${ }^{7}$.

Conforme observado na paciente, a presença de larvas de moscas na UP no calcâneo acabou provocando uma necrose local, dificultando a cicatrização da ferida, pois impedia o crescimento de tecido de granulação. Dessa forma, optou-se por realizar um desbridamento mecânico para viabilizar a proliferação do tecido celular cicatricial e consequente fechamento da ferida.

Casos de miíase no Brasil são comumente relatados na literatura, sendo considerados problema de saúde pública. Tais casos têm relação com o baixo nível social, doenças mentais ou neurológicas, hábitos precário de higiene, pacientes com úlceras varicosas, diabéticos, desnutridos, pacientes com câncer em estágio avançados e os extremos de idade. Alguns autores encontraram em seus estudos evidências de que a maior ocorrência dessa afecção se dá entre mulheres e negros ${ }^{8}$. O presente caso vai ao encontro de tais achados.

Em relação aos idosos que necessitam de cuidados domiciliares, é importante ressaltar que esses podem ser executados por pessoas que possuem ou não vínculo familiar. Pode ser um cuidador formal (capacitado e remunerado) ou informal (que possui vínculo familiar, amigo, vizinho, não sendo economicamente remunerado $)^{3}$. O cuidador informal é o mais comum na maioria das vezes, conforme constatado no presente caso.

A atenção domiciliária, segundo a Política Nacional da Atenção Domiciliar (PNAD) é caracterizada por um conjunto de ações de promoção à saúde, prevenção, tratamento e reabilitação prestadas em domicílio, com garantia de continuidade de cuidados e integrada às Redes de Atenção à Saúde (RAS) ${ }^{9}$. Nesse contexto, pôde-se observar no caso relatado a garantia da integralidade do cuidado à paciente, ao ser referenciada da APS à atenção especializada para desbridamento da ferida infectada, e, após o procedimento, retornar para continuidade dos cuidados na APS. Assim, cabe destacar que para prestar assistência integral e com qualidade, devem ser consideradas as dificuldades relacionadas aos idosos, à própria condição social e o vínculo do idoso com sua família como provedora de condições para a prestação de cuidados ${ }^{10}$.

A assistência domiciliar voltada a esses pacientes requer uma comunicação satisfatória entre a equipe da ESF, o paciente e a família, sendo essa última a principal provedora de cuidados, uma vez que a continuidade dos cuidados fica sob sua responsabilidade ${ }^{5}$. Assim, o papel da ESF inclui o planejamento e implementação de ações que envolvam e valorizem o cuidador, como sendo um novo elemento para a promoção da saúde. Por meio de sua capacitação técnica para manutenção da higiene e mobilização do paciente no leito, os riscos e a prevenção de agravamentos de UPs podem ser minimizados e a morbimortalidade reduzida ${ }^{5}$.

Sabe-se que as UPs têm implicações na relação existente entre a pessoa doente e o familiar, bem como na vida pessoal, familiar e social deste ${ }^{11}$. Assim, para realização das ações de cuidado domiciliar é essencial que sejam consideradas a organização familiar e comunitária em que o paciente está inserido. E ainda, para eficácia do tratamento é fundamental a adesão do paciente e de sua família ao tratamento proposto, já que eles também são responsáveis pelo cuidado ${ }^{12}$.

Para garantir a eficácia do tratamento também é preciso trabalhar com o ambiente e com as pessoas, ressaltar com a família/paciente a importância de promover um ambiente limpo e salubre, com os objetos da casa, executando o manuseio dos mesmos e do próprio paciente de forma asséptica, sempre higienizando corretamente as mãos ${ }^{12}$.

Nesse contexto, por meio das VDs, os ACSs se empenham em promover o autocuidado do usuário, o que inclui o repasse de informações, bem como o aconselhamento sobre práticas e hábitos saudáveis para o paciente, seus familiares e cuidadores ${ }^{13}$. Um plano 
de tratamento adequado inclui ainda o monitoramento das lesões a cada troca de curativo, as quais devem ser reavaliadas, pelo menos, semanalmente ${ }^{14}$, conforme foi feito.

No presente caso, a equipe da ESF foi fundamental ao orientar os cuidadores informais da paciente, no caso a família, a respeito dos cuidados necessários para a eficácia do tratamento instituído. De posse das informações, os cuidadores colocaram em prática as ações que foram essenciais no tratamento da UP.

\section{Considerações finais}

As lesões por UP são consideradas um desafio para assistência em saúde, pois o processo de cicatrização é lento e progressivo e, em geral, as lesões são dolorosas e causam deformidades. Os tratamentos são prolongados e demandam cuidados específicos, e para um fechamento bem sucedido de uma ferida complexa é essencial a realização de um desbridamento bem feito, além de trocas diárias de curativos.

Dessa forma, faz-se mister prestar cuidados integrais aos pacientes com objetivo de garantir a promoção, proteção, recuperação, reabilitação e manutenção da saúde. Uma assistência coordenada aos portadores de UPs, garantindo a continuidade do cuidado integrada à RAS, facilita o acompanhamento dos mesmos, sendo essencial para a eficácia do tratamento. Assim, para os pacientes com UP ou com fatores de risco para as lesões, práticas de cuidado curativo e preventivo eficientes precisam ser implementadas em todos os contextos de atenção.

Cabe destacar ainda que a paresia e também a plegia são alterações que colocam ainda mais em risco os pacientes acometidos por UP. Se esses pacientes não forem referenciados para outros serviços, como os de fisioterapia, por exemplo, para dar continuidade ao processo de reabilitação, o imobilismo poderá levar à formação de novas úlceras.

Medidas preventivas, como ações educativas junto à população e capacitação dos profissionais envolvidos na assistência domiciliária, dos familiares e cuidadores, assim como do paciente, quando possível, de forma que esse problema seja evitado ou amenizado, são estritamente necessárias.

Em suma, para que seja possível a continuidade do cuidado, deve-se iniciar, ainda durante a hospitalização, medidas educativas sobre ações básicas de saúde, como identificar sintomas e complicações das UPs. Assim, é possível proporcionar tratamento e recuperação de forma mais humanizada, gerar bem-estar ao paciente e seu cuidador, além de minimizar a morbimortalidade desencadeada por essas lesões, oferecendo ao paciente a melhor assistência possível em busca de uma melhor qualidade de vida.

\section{Referências}

1. Lima BB, Santos JS. Úlcera por pressão. In: Freitas EV, Miranda RD, Nery MR. Tratado de geriatria e gerontologia. Rio de Janeiro: Guanabara Koogan; 2002. cap.10, p. 1307 - 17.

2. Alcantara CAD, Venícios MOL, Pereira RM, Euridéa MC. O idoso e a úlcera por pressão em serviço de atendimento domiciliar. Rev da Rede de Enfermagem do Nordeste. 2012; 13(3): p.639-49.

3. Ferreira JDL, Aguiar ESS, Lima CLJ, Brito KKG, Costa MML, Soares MJGO. Ações preventivas para úlcera por pressão em idosos com declínio funcional de mobilidade física no âmbito domiciliar. Rev Estima. 2016; 14(1): p.36-42

4. Coqueiro JM, Brito RS. Múltiplos fatores de riscos e estratégias preventivas das úlceras por pressão: revisão sistemática. Rev de enfermagem UFPE On line [Internet]. 2013 [acesso em 22 nov 2018]; 7(10): p. 621522. Disponível em: https://periodicos.ufpe.br/revistas/revistaenfermagem/ article/download/12259/14893.

5. Diniz IV, Soares MJGO, de Aguiar ES, Leite SL. Manejo do enfermeiro em úlceras por pressão infectada no ambiente domiciliar. Rev de enfermagem UFPE On line [Internet]. 2014 [acesso em 22 nov 2018]; 8(1): p. 121-27. Disponível em: https://periodicos.ufpe.br/revistas/ revistaenfermagem/article/viewFile/9614/9592.

6. Bezerra SMG, Barros KM, de Brito JA, Santana WS, Moura ECC, Luz MHBA. Caracterização de feridas em pacientes acamados assistidos pela Estratégia Saúde da Família. Rev Interdisciplinar. 2013; 6(3): p. $105-$ 14

7. Silva PLN, Souza EJ, Fonseca RP, Gonçalves SGTS, Alves ECS, Reis AC, Souza SCL. Importância da comissão de curativos no tratamento das lesões cutâneas: um relato de experiência. Rev Eletrônica Acervo Saúde [Internet]. 2017 [acesso em 12 set 2018]; Vol Sup. 7: p.310-15. Disponível em: https://www.acervosaude.com.br/doc/S-25 2017.pdf.

8. Batista JAS, Moya EFG,Carvalho MMQ. Mí́ases Hu manas Causadas por Larvas de Cochliomyia hominivorax (Coquerel) (Diptera: Calliphoridae) em São Gonçalo, RJ, Brasil: Uma Abordagem Sócio-Econômica. EntomoBrasilis. 2011; 4(3): p.144-46.

9. Pires MRGM, Duarte EC, Gottems LBD, Figueiredo NVF, Spagnol CA. Fatores associados à atenção domiciliária: subsídios à gestão do cuidado no âmbito do SUS. Rev Escola de Enfermagem da USP. 2013; 47(3): p.64856.

10. Moraes GLA, Borges CL, Oliveira ET, Sarmento LR, Araújo PR, Silva MJ. Aplicação de protocolo de prevenção de úlcera por pressão no contexto domiciliar: uma trajetória percorrida. Cogitare Enfermagem. 2013; 18(2): p.387-91.

11. Pereira SM, Soares HM. Úlceras por pressão: perceção dos familiares acerca do impacto emocional e custos intangíveis. Rev de Enfermagem Referência. 2012; 7: p. 139-48.

12. Rocha ACAA, Sales CFA,Souza MS. Tratamento domiciliar de feridas crônicas: relato de experiência da extensão na prática do cuidar. Rev Ciência e Estudos Acadêmicos de Medicina. 2015; 1(2): p.20-30.

13. Magalhães KA, Giacomin KC, Santos WJD, Firmo JO. A visita domiciliária do agente comunitário de saúde a famílias com idosos frágeis. Ciência \& Saúde Coletiva. 2015; 20(12): p.3787-96.

14. Chayamiti EMPC, Caliri MHL. Úlcera por pressão em pacientes sob assistência domiciliária. Acta Paulista de Enfermagem. 2010; 23(1): p.2934 